\title{
p53 Re-Activating Small Molecule Inhibitors for the Treatment of Pancreatic Cancer
}

\author{
Asfar S. Azmi, Minsig Choi and Ramzi M. Mohammad \\ Wayne State University, \\ USA
}

\section{Introduction}

Pancreatic ductal adenocarcinoma (PDAC) devours two American lives every 30 minutes (annual death rate $>37,000$ ) and is the fourth leading cause of cancer-related deaths in the US (Jemal et al. 2010). Median survival is 4 to 6 months and the 5-year survival is less than $5 \%$ (Baxter et al. 2007). The standard chemotherapeutic agent gemcitabine shows dismal response rate and has little impact. Recently, clinicians have incorporated platinum-based genotoxic regimens such as oxaliplatin nevertheless such combinations have little impact on improving the overall survival of PDAC patients (Wang et al. 2011). There are critical unanswered questions regarding the mechanism of drug failure in PDAC and investigations are still a long way from identifying novel drug combination regimens to achieve cure. Therefore, management of PDAC is an ongoing challenge and novel clinically-translatable therapeutic agents that can improve on the dismal survival statistics of PDAC are urgently needed.

To date, PDAC carcinogenesis and drug resistance are only partly understood, due to the heterogeneity of this disease at the cell/tissue level. In general carcinogenesis progresses through the accumulation of genetic alterations resulting in a gain of cell growth and proliferation, and subsequently, in increased dissemination and metastatic potential and PDAC is not an exception (Whitcomb and Greer 2009). Loss or gain of gene function may appear in the form of up-regulation of oncogenes, down-regulation of tumor suppressor genes, and deregulation of genomic maintenance/DNA repair genes, house-keeping genes, and genes that control the apoptosis/cell death/immortalization cascade (Sohn 2002; Hruban et al. 2007; Hruban et al. 1999). PDAC arises from precursor lesions called pancreatic intraepithelial neoplasms (PanINs), which are characterized by the sequential accumulation of alterations in the K-ras oncogene and loss of the CDKN2A, TP53 (p53), and/or SMAD4 tumor suppressors (Hruban et al. 2000). Although we know the frequencies of such mutations in PDAC, their specific functions during the development of PDAC remain unclear. K-ras and p53 are considered to be among the most critically deregulated genes that participate in cross talk to render PDAC therapy resistance (Hollstein et al. 1998; Hollstein et al. 1994). In the forthcoming passages we will first describe the impact of de-regulated p53 signaling on therapy resistance and then update on the pharmaceutical progress of small molecule inhibitors against p53 and related targets for the treatment of PDAC. 


\section{Impact of dysfunctional p53 signaling on PDAC therapy resistance}

p53 tumor suppressor gene is mutationally inactivated in $>50 \%$ of PDAC (Hohne et al. 1992; Kalthoff et al. 1993), predominantly through missense mutations (Pellegata et al. 1994). These often result in accumulation of mutant p53 protein, with potentially gain-of-function or dominant-negative properties. The fact that p53 is mutated, rather than deleted, in the majority of PDAC suggests that mutant p53 provides some tumor cell growth advantage. Murine models support this as mice expressing the accumulating p53 mutants p53R $172 \mathrm{H}$ or p532270H have increased incidence of osteosarcoma and epithelial carcinomas, some of which spread to distant organs (Lang et al. 2004; Olive et al. 2004). In contrast, mice that harbor a p53 null allele rarely develop metastases. It has also been recognized that mut-p53 and family member p63 protein can inhibit wt-p53 function, indicating that bi-allelic inactivation may not be necessary for loss of function. The third family member, p73, is rarely mutated and has been shown to possess apoptotic function in response to small molecule inhibitors and platinum drugs-induce apoptosis. Furthermore, in the other 50\% of PDAC, the p53 gene is normal (wild type wt-) but its function is inhibited by MDM2 (human double minute 2); a protein that is over expressed in PDAC and is the primary focus of this chapter.

The activity of wt-p53 is mainly regulated at the post-translational level through its proteolytic turn over (Brooks and Gu 2006). This is achieved through the interaction with MDM2, which induces wt-p53 degradation by ubiquitin-mediated proteolysis (Bottger et al. 1997). In normal, non-stressed cells, MDM2 induces p53 degradation constantly, making it a short-lived protein. However, in response to DNA damage, MDM2 is auto-polyubiquitinated, resulting in its degradation and an associated increase in p53 levels and activity. This regulatory mechanism is subject to a feedback loop since p53 in turn, regulates the level of MDM2 transcription, giving rise to subtle balances between the amounts of p53 and MDM2 (Fig1) (Lahav 2008; Bose and Ghosh 2007). Although other isoforms of MDM2 are also present, i.e. HDM4 (Mancini et al. 2009a; Okamoto et al. 2009; Mancini et al. 2009b), but carry have lesser impact on p53. Despite being an energy consuming process, the MDM2-post-translational regulation of the amount of p53 is advantageous for cells because it is rapid and it increases the odds of having functional p53 following genotoxic stress and is in contrast to transcriptional regulation which is slower and sensitive to DNA damage (Millau et al. 2009). A common $M d m 2$ promoter polymorphism is the $\mathrm{T} \rightarrow \mathrm{G}$ transformation at nucleotide 309. This $M d m 2309 T / G$ promoter polymorphism has been associated with the development of a variety of tumors including PDAC (Grochola et al. 2010b; Bond et al. 2004; Ohmiya et al. 2006b; Galic et al. 2007; Lind et al. 2006). Its significance in clinical outcome is not well outlined, but recent associations with prognosis have been found in lung and gastric cancer (Ohmiya et al. 2006a). The G allele is associated with increased affinity for Sp1 binding and higher MDM2 mRNA and protein levels, leading to diminished tumor suppressor activity of the p53 pathway (Iwakuma and Lozano 2003). Over-expression of MDM2 in cancers prevents this normal balance and thus inhibits p53 even though it may not be mutated (bdel-Fattah et al. 2000; Watanabe et al. 1994; Watanabe et al. 1996). Based on functional significance of MDM2 in the biology of p53, one attractive pharmacological approach to wt-p53 activation is to use a small molecule weight inhibitor (SMI) to block the MDM2-p53 interaction (Klein and Vassilev 2004; Secchiero et al. 2008; Vassilev 2004b). Further, it is well recognized that genotoxic drugs such as oxaliplatin work through activation of p53 signaling, however, mutations in the gene or over-expression of MDM2 is 
logical to thwart proper p53 response and therefore diminish the therapeutic potential of these drugs. Thus, re-activation of p53 by blocking MDM2 is an attractive pharmacological approach for treatment MDM2 over expressing subtype of PDAC (Azmi 2011; Azmi et al. 2010c). Nevertheless, there are additional de-regulated signaling molecules and p53 masking mechanisms that come into play. It is the lack of understanding of these regulatory control mechanisms that had led to failure in the effective design of drugs. As discussed below, the success of novel drugs is possible if the proper combination is identified that hits each component of the PDAC resistant network to achieve clinically beneficial outcome.
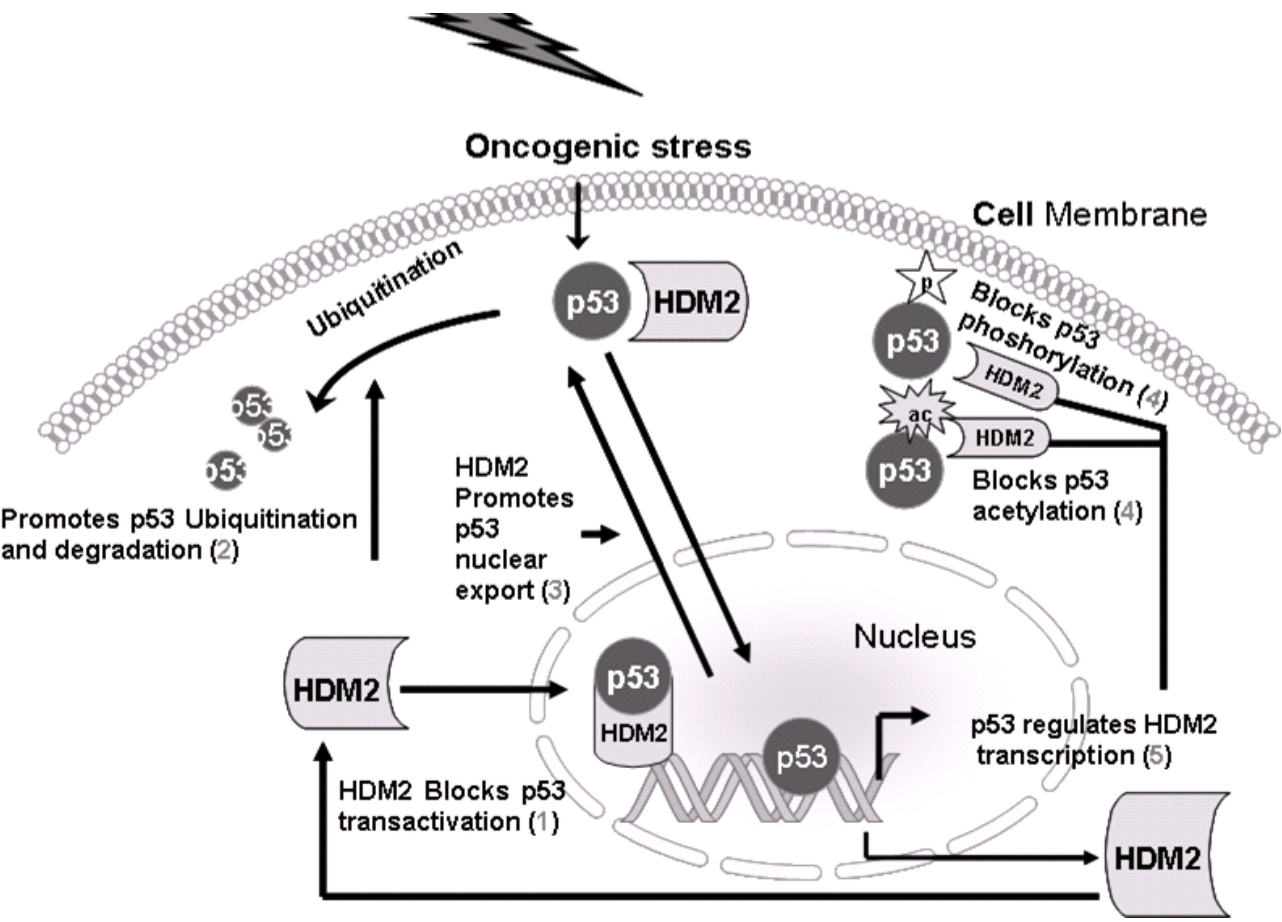

Fig. 1. MDM2 is a Master Regulator of p53: MDM2 blocks p53 activity at multiple levels. (1) MDM2 can bind directly to p53 activation domain and suppress its transcription. (2) MDM2 acts as E3 ligase and promotes p53 proteasomal degradation. (3) MDM2 has a nuclear export signal sequence in its domain structure that is responsible for p53 nuclear export. Cytoplasmic retention of p53 suppresses its nuclear transactivation activity. (4) MDM2 can block post-translational modifications of p53 (acetylation, phosphorylation etc necessary for p53 activity). (5) In turn, the transcription of MDM2 is regulated by p53. (Adopted from Azmi et al. 2011)

\subsection{Regulation of p53 by hedgehog driven K-ras-snail axis}

PDAC is an oncogenic K-ras driven disease and it is well established that majority of PDAC tumors show alteration in this pathway (Laghi et al. 2002). Although 3 kinds of oncogenic Ras have similar roles in induction of cell proliferation, survival, and invasion through the 
stimulation of several pathways (Downward 2003), the mutation rate of K-ras is relatively higher than others, which indicates that the genetic alteration of K-ras is a major driving force for PDAC. Recently, the regulatory network of mutant K-ras signaling on p53 pathway has been established. Studies indicate that in cancer, p53 activity is suppressed via a novel mechanism of K-ras activation and consequent stabilization of snail (Fig2).

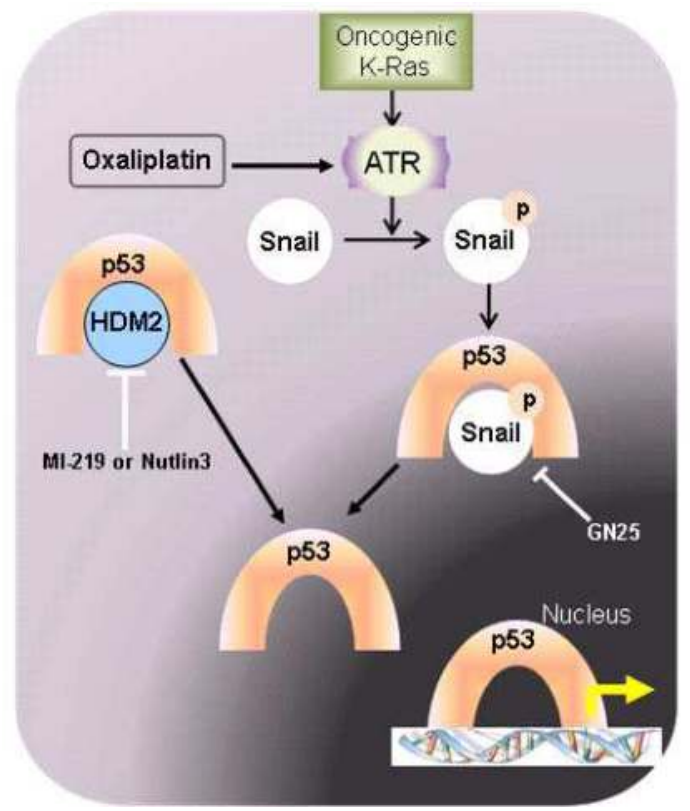

Fig. 2. Schematic diagram showing snail-p53 interaction that suppresses therapeutic response of genotoxic p53 re-activating regimens. Snail is activated through oncogenic K-ras driven ATR pathway while genotoxic regimens additionally induce snail. Novel agents targeting snail can have therapeutic implications against K-ras driven PDAC.

Snail stabilization occurs through activated ATR in K-ras mutated cells that demonstrates a strong direct interaction between snail and DNA-binding domain of p53, resulting in elimination of both protein from the cells (Horiguchi et al. 2009). This novel regulatory network has been shown to be independent of MDM2 or ARF-mediated p53 regulation. In normal cells, activation of oncogenic K-ras has been shown to provoke apoptosis or senescence through p53 activity, indicating that the loss of functions through p53 mutation is inevitable for the tumor progression by oncogenic K-ras (Gorgoulis and Halazonetis 2010). In mouse models, the adenoma is evoked by oncogenic K-ras despite the intact p53 system, which highlights that the additional and unique function of K-ras may enable cancer cells to avoid the tumor suppressive function of p53. Recent studies have also demonstrated the apparent tumorigenic function of oncogenic K-ras in mouse cell transformation (White et al. 2011). While normal mouse fibroblast transfected with $\mathrm{N}$ - or H-ras induce apoptosis or senescence, K-ras12V transfected cells become resistant and this is consistent with the multistep carcinogenesis model of colon or PDAC. In fact, genetic mutations of K-ras occur in the early adenoma stage without p53 mutation. However, large portion of adenoma can 
be progressed into carcinoma without impaired p53 system or consequent apoptosis or senescence. These observations strongly indicate that oncogenic K-ras-snail axis possesses strong p53 suppressive effects downstream of MDM2 that cannot be ignored. Additionally, hedgehog signaling that is considered driver for PDAC induces Gli that in-turn induces snail adds to the existing ATR-driven suppressive mechanisms (Marigo et al. 1996). Thus, strategies that overcome these inhibitory pathways need to be incorporated in any genotoxic regimen that targets activation of p53 system. In sum, these findings demonstrate that in addition to MDM2 suppression targeting p53-snail binding can bring additional benefit to genotoxic therapy to achieve clinically superior therapeutic effects in PDAC.

Because the interaction between MDM2 and p53 is a primary mechanism for inhibition of the p53 function in cancers retaining wild-type p53, targeting the MDM2-p53 interaction by small molecules to reactivate p53 has emerged as a promising new cancer therapeutic strategy. In this chapter, the emerging SMIs of MDM2-p53 interaction are evaluated with additional discussions on new snail-p53 interaction inhibitors for the treatment of pancreatic cancer.

\section{Reactivating the wild-type p53}

Inhibition of p53 activity in tumors by the increased expression of MDM2 has been the target of development for many small-molecule-, peptide- and aptamer-based therapies (Lehman et al. 2008). MDM2 is over expressed in many human tumors including PDAC, often owing to an amplification of a chromosome segment that includes $M d m 2$, although over expression of the protein is possible without gene amplification (Do et al. 2009; Assmann et al. 2009; Shinohara et al. 2009; Grochola et al. 2010a; Lang et al. 2009; Economopoulos and Sergentanis 2009; Perfumo et al. 2009). There has been extensive validation of MDM2 as a target, ranging from studies with aptamers and peptides through to antisense approaches and, perhaps most significantly, was described in a path breaking study using a hypomorphic allele of $M d m 2$ in the mouse (Mendrysa et al. 2003). In this study, nominal reductions in MDM2 levels were found to be sufficient to trigger a mild p53 response (as shown by increased levels of lymphopenia and apoptosis in intestinal crypts) in response to increased p53 activity. The volume of the thymus is also reduced and there is a small effect on weight gain during development. Gene dosage studies have found levels of MDM2 that selectively inhibit the development of colon carcinoma induced by the absence of adenomatous polyposis coli $(A P D A C)$ without adverse affects on normal tissues. These powerful studies provided proof of a therapeutic index for MDM2 inhibition that has now been confirmed by the first small molecule candidates, including Nutlin (Vassilev et al. 2004), MI-219 (Shangary and Wang 2009) and reactivation of p53 and induction of tumor cell apoptosis (RITA) also known as NSC 652287 (Hedstrom et al. 2009), which produce tumor regression in vivo in human tumor xenografts in nude mice. These initial studies paved way for large scale investigations on different types of MDM2 inhibitors and are discussed below.

\subsection{Non-peptidic small-molecule inhibitors of MDM2-p53 interaction}

The progress in the design of nonpeptidic, small-molecule inhibitors of the MDM2-p53 interaction (mentioned herein as MDM2 inhibitors; sometimes also called as MDM2i inhibitors) proceeded very slowly for almost a decade after the publication of the crystal 
structures. The very first class of bona fide, potent, nonpeptidic, small-molecule MDM2 inhibitors, known as Nutlins, was reported in 2004 (Vassilev 2007; Vassilev 2005; Vassilev 2004a). The Nutlins contain a cis-amidazole core structure and one analogue, Nutlin-3, has potent in vivo antitumor activity in xenograft models of human cancer-retaining wild-type p53. The discovery of the Nutlins provided the important proof-of-concept and fueled enthusiasm for the design and development of small-molecule MDM2 inhibitors. In the last 4 years, several new classes of small-molecule MDM2 inhibitors have been discovered using different approaches (Canner et al. 2009; Shangary et al. 2008a; Fotouhi and Graves 2005). Using a computational structure-based de novo design strategy, a new class of spirooxindoles that are potent inhibitors of MDM2 (Ding et al. 2006), as exemplified by MI-63 and MI-219 were designed. In this regard Nutlin-3 a cis-imidazole has been well studied in different cancers. Our MI series of MDM2 inhibitors belong to different class (spirooxindole) and have a slightly higher affinity towards MDM2 when compared to Nutlins. Using a structure-based de novo design strategy it was shown that the interaction between p53 and MDM2 is primarily mediated by four key hydrophobic residues (Phe 19, Leu 22, Trp 23 and Leu 26) of p53 and a small but deep hydrophobic cleft in MDM2. Nutlin-3 mimics the interactions of the p53 peptide to a high degree, with one bromophenyl moiety sitting deeply in the Trp pocket, the other bromophenyl group occupying the Leu pocket, and the ethyl ether side chain directed toward the Phe pocket. In essence, the imidazoline scaffold replaces the helical backbone of the peptide and is able to direct, in a fairly rigid fashion, the projection of three groups into the pockets normally occupied by Phe19, Trp23, and Leu26 of p53. However, unlike Nutlin-3 in case of our inhibitors (MI series), computational modeling predicted that MI-219 mimics the four (instead of three in case of Nutlin-3) key binding residues in p53 (Phe-19, Leu-22, Trp-23 and Leu-26) resulting in optimal hydrogen bonding and hydrophobic interactions with MDM2. Both Nutlins and MI-219 enter many types of cultured cells and inhibit the p53-MDM2 interaction with a high degree of specificity, leading to the stabilization of p53 and the activation of the p53 pathway. Proliferating cancer cells that express wild-type p53 are effectively arrested in the G1 and G2 phases of the cell cycle or can undergo apoptosis when treated with micro molar concentrations of Nutlins (Shangary et al. 2008b). This indicates that some cells are more susceptible to Nutlin-induced apoptosis than other cells in which a reversible cell cycle arrest is observed. The key cellular characteristics that underlie this difference in response are the subject of intense investigation. This differential response may occur owing to abnormalities further downstream in the p53 pathway. Other small molecules that have been developed to target the p53-MDM2 interaction include benzodiazepenes (Patel and Player 2008). The benzodiazepene-based derivatives disrupt the MDM2-p53 interaction in vitro with $\mathrm{IC}_{50}$ values of $0.5-2 \mu \mathrm{M}$ and have also been shown to suppress the growth of cell lines containing wild-type p53. Administration of the benzodiazepene derivative TDP665759 to normal mice led to an increase in p21 (also known as WAF1 and CIP1) levels in liver samples (Koblish et al. 2006). Finally, TDP665759 synergizes with doxorubicin both in culture and in xenografts of A375 melanoma cells to decrease tumor growth.

With so many targets identified against the MDM2-p53 interaction and supporting preclinical laboratory evidence it is imperative that effective MDM2 inhibitors will become a major form of therapy in the coming years. However, several potential drawbacks to targeting the MDM2-P53 interaction can be envisioned. First, MDM2 is induced by p53 activation as part of an inducible feedback loop that negatively regulates the p53 response. 
Therefore, the drugs would induce their target, limiting their potential efficacy. Second, the current molecules fail to effectively target MDM4. The binding pocket of the $\mathrm{N}$ terminus of MDM2 has shown itself to be eminently druggable, and a future challenge is whether or not these drugs can proceed to the clinic and whether they can also be refined to target other MDM2 family members such as MDM4. Apart from this avenue of research, other target sites have been identified in this p53 regulatory pathway that show the potential for drug development, and it remains to be seen if they generate therapeutic leads that have low toxicity in normal tissues.

\subsection{Compounds that target $\mathrm{p} 53$ regulators}

Activated p53 is under multiple post-translational control that includes acetylation, methylation, phosphorylation, neddylation and sumoylation (Lee and Gu 2010; Kruse and Gu 2009; Halaby and Yang 2007; Schumacher and Gartner 2006; Chuikov et al. 2004; Haupt 2004; Brooks and Gu 2003; Wiederschain et al. 2001; Somasundaram 2000; Craig et al. 1999). Activating p53 using small may not be sufficient for proper p53 function and combinations with agents that suppress post-translational p53 blockers such as acetylation would benefit the overall outcome of such therapy. An example of this type of agent which was identified through a p53-based phenotypic screen are tenovin-1 and its more water-soluble derivative tenovin-6 (Lain et al. 2008; Brooks and Gu 2008). Tenovins rapidly increase p53 levels in cells treated with low micro molar concentrations, and daily intraperitoneal injection of tenovin-6 at $50 \mathrm{mg}$ per kg delays xenograft tumor growth in mouse models. Through a yeast genetic screen and subsequent enzymatic assays tenovins were shown to inhibit the $\mathrm{NAD}^{+}$ dependent deacetylase activity of SIRT1 and SIRT2 (Canto and Auwerx 2009; Campisi and Yaswen 2009), two members of the sirtuin family of class III histone deacetylases. p53 deacetylation by SIRT1 impairs p53 stability and transcriptional activity. Therefore, inhibiting the sirtuins should lead to increased p53 stability. Indeed, treatment of MCF-7 cells with tenovins led to the accumulation of acetylated p53 and acetylated tubulin, which are established substrates of SIRT1 and SIRT2, respectively. Further chemical optimization of the potency of the tenovins is now possible owing to the elucidation of SIRT1 and SIRT2 as the cellular targets. The discovery and characterization of the tenovins is an example of how current technological advances in target identification and p53 basic research contribute to the understanding of the mechanism of action of bioactive small molecules.

\subsection{Snail-p53 interaction inhibitors}

Rapid progress has been made in the development of novel chemicals which can block the K-Ras-mediated p53 suppression through the screening of the chemical library. Using ELISA-based chemical screening for discovering potent chemical inhibitors that are effective to prevent the binding were discovered. Interestingly, one group of the chemical library particularly showed high effects in blocking the interaction of Snail and p53 (Lee et al. $2010 b$ ). Since this chemical library was arranged in accordance with its chemical structural similarity, it strongly implies that the similar structural motif of chemicals can impact p53snail interaction. Among the compounds of this group, B3 and C2 were selected as candidates that could effectively provoke p53 and its target genes, such as p21 and PUMA, in K-Ras mutated cancer cell lines (Lee et al. 2009a). GN25 and GN29 has been revealed to show substantial results that induce p53 and p21 expression, as much as Nutlin-3 (small 
molecule inhibitor that specifically blocks p53-MDM2 binding). Strong candidates (GN25 and GN29) can activate the p53 in K-Ras mutation dependent manner (Lee et al. 2010a) (Fig 2). GN chemicals show similar activity on p53 activation only in K-Ras mutated cell. Moreover, GN25 shows no cytotoxic effect in normal or K-Ras wild type cancer cells.

Of importance is the observation that GN chemicals can induce p53 and p21 occurred as strong as in the treatment of DNA damaging agents such as adriamycin or etoposide, and the anti-proliferating effect of GN chemicals was even stronger than Nutlin-3. In addition, in normal fibroblast, any significant differences not only on the p53 expression level, but also on its proliferation and viability was not detectable by GN chemicals, but by Nutlin-3, which reveals that GN chemicals possess the powerful and specific property unique to other chemicals. The extensive duration of the p53 activity response to low concentration of GN25 and GN29 was shown for over 24 hours, which indicate that these chemicals successfully remedy problem of very short re-activation of p53. One crucial fact is that another additional derivative, GN25-1 containing the same side chain but a modified nuclear structure of GN25, has no effect on the induction of p53, which suggest that the conserved nuclear structure of the GN chemical is critical in increasing p53 activity.

In the condition of disrupted Snail, p53 induction could be shown in K-Ras mutated cancer cells, but there is no synergic and additional effect by GN25 and 29, which clearly indicate that p53 induction by GN25 and 29 can occur through interfering Snail-p53 binding. To identify the specificity of these chemicals on Snail-p53 binding, several surrounding factors involved in the p53 function should be checked. Since the middle region of Snail is normally targeted for CK1/GSK3 $\beta$-dependent phosphorylation (Cano et al. 2000). In addition, although p53 has no influence on $\beta$-Catenin phosphorylation by CK1/GSK3 $\beta$, recombinant p53 sufficiently interferes with CK1/GSK3 $\beta$-mediated Snail phosphorylation (Yook et al. 2006). Indeed, quercetin (inhibitor of Snail-p53 binding) and GN25 could recover CK1/GSK$3 \beta$-mediated Snail phosphorylation even in the presence of p53 (Lee et al. 2009b). In contrast, GN25 did not affect the physical interaction of p53 with other proteins such as WRN making it a p53 specific targeted SMI (Blander et al. 1999).

One of very exciting feature is that GN25 and GN29 block the interaction between Snail with wild type p53 but not with mutant type p53. This is resulted from the property of chemicals, which can bind to wild type p53. Indeed, GN chemical can rescue the only wild type p53 in wild and mutant p53 expressed cells from K-Ras or Snail-mediated p53 suppression. Thus, GN chemical can work in p53 mutated cancer cells, if it possesses single copy of p53. In fact, although GN25 and 29 decrease total p53 expression level in p53 WT/MT cells (no effect on the transcriptional level), it can induce p21 and PUMA and reduce the proliferation and viability of cells. This result was not accomplished by other DNA-damaging agents including Adriamycin or Nutlin-3. By the use of GN chemicals, all of the p53 proteins might be protected from Snail-mediated elimination in p53 WT/WT status. These features of GN chemical can extend the application range from p53 wild type cancer to p53 mutated cancer and from early cancer to late stage cancers.

\section{MDM2 inhibitor investigations in PDAC}

Testing of MDM2 inhibitors have been restricted in PDAC and this may be due to lack of suitable cell lines (Capan-2 is the only wt-p53 PDAC cell line). To this end our laboratory is 
the first to show the growth inhibitory and apoptotic potential of MDM2 inhibitors MI-319, MI-219 and Nutlin-3 in PDAC (Azmi et al. 2010b). These inhibitors specifically induced cell growth inhibition and apoptosis in wt-p53 PDAC cells. Growth inhibition and apoptosis by MDM2 inhibitors was accompanied by increase in levels of p53 along with p21WAF1 and the proapoptotic Puma. In these studies immunoprecipitation-western blot analysis revealed reduced association of MDM2-p53 interaction in drug exposed PDAC cells. Further, using wt-p53 xenograft of Capan-2, we found that oral administration of MI-319 at $300 \mathrm{mg} / \mathrm{kg}$ for 14 days resulted in significant tumor growth inhibition without any observed toxicity to the animals. No tumor inhibition was found in mut-p53 BxPC-3 xenografts. We also explored combination treatments of these inhibitors with chemotherapeutics such as gemcitabine and cisplatin. Interestingly MI-319, MI-219 combination specifically enhanced cell growth inhibition and apoptosis in PDAC cells with wt-p53 suggesting that this potent combination can be used in the clinic for wt-p53 population in this dreadful disease. We also tested the combination of MI-319 with cisplatin which although less popular for PDAC yet is a potent p53 inducer. Apart from being a potent combination in wt-p53 PDAC tumors most interestingly MDM2 inhibitor-cisplatin combination drastically induced growth inhibition apoptosis and tumor growth inhibition in mut-p53 PDAC cell lines and xenografts respectively (Azmi et al. 2010a). Mechanistically using siRNA silencing it was proven that the p53 family member p73 (which is rarely mutated in PDAC and other cancers) was found to be responsible for the observed anticancer/anti-tumor effects. That the combination worked in a p53 null system (HCT116--) further reiterated that MI-319-cisplatin combination could effectively induce apoptosis through a p73 dependent mechanism. However, further in-depth mechanistic studies are warranted specifically in PDAC.

The availability of potent and specific MDM2 inhibitors, such as Nutlin-3 and MI-219, has provided the opportunity to examine in detail the molecular mechanism of p53 activation. In competition with a p53-based peptide, Nutlin-3 and MI-219 bind with high affinity to MDM2 $\left(K_{i}=36\right.$ and $5 \mathrm{nmol} / \mathrm{L}$, respectively). They block the intracellular MDM2-p53 interaction and induce the accumulation of p53 and the activation of the p53 pathway in tumor and normal cells. Conventional genotoxic anticancer agents and radiation also induce the accumulation and activation of p53, but they do so by posttranslational modifications of p53, such as phosphorylation. In contrast, Nutlin-3 induces neither DNA damage nor p53 phosphorylation in cells. Hence, small-molecule MDM2 inhibitors represent a new class of non-genotoxic agents that can reactivate the p53 function. Apart from regulating p53 recent studies have shown p53 independent functions of MDM2. In this regard Zhang and coworkers have extensively reviewed a whole range on targets governed by MDM2 (Zhang and Zhang 2005). Targeting MDM2 by agents such as MI-219 or Nutlin may affect a myriad of other key cellular molecules that play significant role in cell growth and apoptosis. Indepth mechanistic studies on the mode of action of inhibitors on MDM2 and the consequent p53 reactivation are lacking and it was of interest to us to explore the roles of crucial proteins that are involved in the regulation of p53. Activated p53 is known to be influenced by multiple post-translational control processes such as phosphorylation and acetylation that positively regulate p53 function (Stommel and Wahl 2005). Acetylation is an important epigenetic phenomenon in the biology of p53 (Gu et al. 2004; Ito et al. 2002). Upon stress, p53 is acetylated at Lys382 which enhances its DNA binding activity (Hasegawa and Yoshikawa 2008). Moreover, deacetylation of p53 by SIRT1 has been shown to repress p53 mediated cell cycle regulation and apoptosis. SIRT1 is also known to deacetylate another 
protein Ku70 which, in turn, interacts with Bax and is responsible for blocking Bax entry into mitochondria. Therefore, we sought to determine whether acetylation of p53 could be influenced by our inhibitors in PDAC cells. Indeed our results showed that MI-219 treatment suppresses SIRT1 protein and simultaneously enhances acetylation of p53. Using state of the art Surface plasmon resonance techniques we studied the binding between MI319 or Nutlin-3 and Ku70 and our results confirm high affinity association between the two. Interestingly MI-219 treatment resulted in the suppression of Ku70 expression along with disruption of Ku70-Bax interaction. This observation is of great importance because it proves that MDM2 inhibitor not only blocks MDM2 which is its primary target but also suppresses two secondary targets the negative regulator 'SIRT1', which is a molecule that regulates p53 function and $\mathrm{Ku} 70$. Although it is too preliminary to confirm the true binding/interaction site of Ku70 or SIRT1 to MI-319, yet is can be speculated that MI-319 or Nutlin-3 may interact with peptide sequence (LSQETFSDLWKLL) similar to p53 transactivation domain towards which both Nutlin-3 or MI series of inhibitors were built.

As MI-219 does not alter MDM2 expression yet Ku70 and SIRT1 are suppressed suggesting that these drugs may have a MDM2 independent role in the biology of cells. However, compelling evidence in literature supports to a MDM2 dependent mechanism of action of these drugs on Ku70 and SIRT1. Our cell free FRET based SIRT1 activity assay showed inhibition of SIRT1 activity by MDM2 inhibitors. Yet in a cellular system the dynamics of SIRT1 is complex. Studies so far suggest that only wt-p53 can inhibit SIRT1 while cells that have lost or have mutations in p53 have over expressed SIRT1 and cannot repress it. This certainly points out that the suppression of SIRT1 in our system is p53 dependent however elucidation of the exact mechanism of action requires further work. As far as Ku70 is concerned, very recently Nutlin, a drug with similar mode of action as MI-219 was shown to disrupt MDM2-Ku70 interaction. Based on our results and those of others we propose multiple mode of action MI-219 on SIRT1 and Ku70. MDM2 inhibitors down regulate SIRT1 that in principle may prevent Ku70 and p53 deacetylation. Surface plasmon resonance and Co-IP results confirm that MI drugs directly bind to Ku70 as well as disrupt Ku70-Bax interaction. Although yet to be proved, it is suggested that such a direct binding may induce conformational changes in Ku70 rendering it ineffective in binding to Bax and therefore allowing the latter to induce apoptosis. MI drugs also directly suppress Ku70 mRNA and protein expression which in turn allows p53 induced free Bax to mediate apoptotic events.

Additionally, we have utilized a systems biology and network modeling approach to investigate in mechanistic detail the mechanism of action of MDM2 inhibitor and its oxaliplatin combination in PDAC (Azmi et al. 2010d; Azmi et al. 2011). Microarray profiling of a wt-p53-containing PDAC cell line (Capan-2) treated with either MI-219, oxaliplatin, or their combination, revealed some very interesting results that may have clinical implications. Global analysis of genes showed that MI-219 treatment resulted in the alteration of only 48 genes, which highlights the targeted nature of MDM2 inhibitor MI-219. On the other hand, oxaliplatin is a cytotoxic agent and caused alteration of 761 genes. The combination of MI-219 with oxaliplatin resulted in 767 genes being altered. The most important aspect of this finding is the emergence of 286 synergy-specific unique genes that were not found in the MI-219 alone or in the oxaliplatin-treated group. This finding confirms that the synergy between MI-219 and oxaliplatin is at the gene level. Principle component analysis showed that the global gene signatures between single treatments 
versus combination treatments were non-overlapping and could be differentiated at different time points. Molecular network modeling of a total of 767 gene-associated pathways revealed a total of 22 statistically enriched functional groups that were linked to biologically distinct functional pathways. Interestingly, network modeling of the 286 synergy-unique genes showed statistical enrichment of 14 disease (cancer) relevant pathways. This finding suggests that these pathways are relevant to cancer, further indicating that the combination synergy between MI-219 and oxaliplatin is at the gene level, comprising distinct biologically meaningful processes. Further analysis of the combination treatment network revealed the presence of several local networks, or hubs, rather than a single hub of activity interconnecting MDM2-p53. Central players such as the CREB binding protein (CREBBP; i.e., ubiquitously expressed gene) that is involved in the transcriptional coactivation of many different transcription factors, including p53, collaborates/cooperates with ARF (CARF) that is responsible for p53 stability, and NF-KB and early growth response protein (EGR1) tumor suppressor module, all of which are known to positively affect p53 reactivation, which in principle would drive cells toward increased apoptosis. Most importantly, these observed gene changes could also be validated at the mRNA and protein level.

Other investigators have also performed expression signature analysis of Nutlin-3. For example gene expression profiling of Nutlin-3 has been done by Zauli and group in B-cell lymphocytic leukemia (B-CLL) (Zauli et al. 2009). In their study B-CLL patient samples were exposed to Nultin-3 and cDNA expression profiling was performed. With the exception of a few cases, the authors noted induction of a characteristic gene expression profile (GEP) signature that was similar in the majority of B-CLL patient samples. Most significantly, lack of characteristic signatures correlated with poor response to Nutlin-3. However, partial lack of response in these wt-p53 B-CLL samples was not due to defects in the ability of Nutlin-3 to promote p53 induction, but suggested the involvement of secondary masking mechanisms. Nutlin-3 gene signatures were all related to the p53 network and included downstream affector genes such as Fas and Bax and activation of auto-regulatory MDM2. Such type of biological analysis, if coupled with network modeling may provide further information on the entire set of genes modulated by Nutlin-3 in B-CLL patients. Results of these studies will significantly aid in the design of clinically successful drug combinations for other malignancies, which will ultimately benefit the overall survival of patients irrespective of the mutational and functional status of p53. In another study, a large-scale RNA interference-based short hairpin RNA (shRNA) barcode screen was applied to gain insight in the mechanism of action of Nutlin-3 (Brummelkamp et al. 2006). In this study it was shown that aside from p53, 53BP1 was critical mediator of Nutlin-3-induced cytotoxicity. 53BP1 is part of a signaling network induced by DNA damage that is frequently activated in cancer but not in healthy tissues (DiTullio, Jr. et al. 2002). These results suggest that tumor specificity of Nutlin-3 may result from its ability to turn a cancer cell-specific property (activated DNA damage signaling) into a weakness that can be exploited therapeutically.

\section{Current status of MDM2 Inhibitors in the clinic}

Although proven to be successful in the laboratory in multiple cancer models, MDM2 inhibitors or approaches that utilize reactivation of p53 have a long way to go before they 
are acceptable in the clinic for PDAC. Currently some SMIs that reactivate the mut-p53 through protein conformational changes are currently in Phase I clinical trials (Brown et al. 2009). In addition to the potential clinical applications, discovery of the first MDM2 antagonist, Nutlin-3a provided the initial proof of concept that inhibition of protein-protein interactions was a feasible approach to pharmaceutical design. Since that time, a deluge of patents and reports have disclosed a high number of diverse molecules showing potency and selectivity toward MDM2 at the same time lacking in solubility. Careful attention to medical chemistry was employed to improve bioavailability of these scaffolds leading to compounds with optimized PK properties. The intense labor of research has finally begun to bear fruit as demonstrated by the advancement of JNJ-26854165 and RG7112, into early phase clinical trials. Preliminary data from trials of both compounds suggest potential for advancement to Phase II trials and beyond. A few examples are PRIMA (Phase I APR-246), CP-31398 (Phase I) and PhiKan-08 (Phase I). SMIs that activate p53 through disruption of MDM2-p53 binding such as MI-219, Nutlin-3 are in phase I. RITA a p53 binding targeted agent, tenovin (SIRT1 inhibitor are still in a pre-clinical testing phase. Leptomycin B (a CRM1 (Exportin 1) binding agent that mediates p53 reactivation) is in Phase I while Actinomycin D (an RPL11 and RPL5 (Ribosomal protein L) releasing agent) has been approved for Phase I (Choong et al. 2009). Certain combinations such as nutlin with mitotic inhibitors for example BI-2536 (PLK1 (Polo-Like Kinase) inhibitor) or with VX680 (Aurora kinase inhibitor) are also in Phase I.

\section{Conclusion}

In PDAC, multiple de-regulated signaling especially the MDM2 over-expression and hyper activated K-ras driven pathways hone in on p53 and suppress its proper function in controlling various cellular processes. Studies indicate that these suppressive mechanisms render p53 re-activating genotoxic therapies ineffective. The well-studied role of p53 in coordinating cellular response to stress, aberrant growth signals and genomic instability has established a solid rationale for the targeting of MDM2 and K-ras driven snail to restore therapeutic response to treatment of PDAC. The entry of orally-administered MDM2 antagonists into clinical trials represents a significant advancement for the field of small molecule drug discovery in PDAC.

\section{Acknowledgment}

The authors acknowledge the grant support from National Cancer Institute (NIH) R01CA109389 to R.M. Mohammad)

\section{References}

Assmann G, Voswinkel J, Mueller M, Bittenbring J, Koenig J, Menzel A, Pfreundschuh M, Roemer K and Melchers I. (2009). Clin Exp Rheumatol, 27, 615-619.

Azmi AS. (2011). Curr Pharm Des, 17, 534-535.

Azmi AS, Aboukameel A, Banerjee S, Wang Z, Mohammad M, Wu J, Wang S, Yang D, Philip PA, Sarkar FH and Mohammad RM. (2010a). Eur J Cancer.

Azmi AS, Beck FW, Sarkar FH and Mohammad RM. (2011). Curr Pharm Des, 17, 640-652. 
Azmi AS, Philip PA, Aboukameel A, Wang Z, Banerjee S, Zafar SF, Goustin AS, Almhanna K, Yang D, Sarkar FH and Mohammad RM. (2010b). Curr Cancer Drug Targets, 10, 319-331.

Azmi AS, Philip PA, Almhanna K, Beck FW, Sarkar FH and Mohammad RM. (2010c). Mini Rev Med Chem, 10, 518-526.

Azmi AS, Wang Z, Philip PA, Mohammad RM and Sarkar FH. (2010d). Mol Cancer Ther, 9, 3137-3144.

Baxter NN, Whitson BA and Tuttle TM. (2007). Ann Surg Oncol, 14, 1320-1326.

bdel-Fattah G, Yoffe B, Krishnan B, Khaoustov V and Itani K. (2000). J Gastrointest Surg, 4, 109-114.

Blander G, Kipnis J, Leal JF, Yu CE, Schellenberg GD and Oren M. (1999). J Biol Chem, 274, 29463-29469.

Bond GL, Hu W, Bond EE, Robins H, Lutzker SG, Arva NC, Bargonetti J, Bartel F, Taubert H, Wuerl P, Onel K, Yip L, Hwang SJ, Strong LC, Lozano G and Levine AJ. (2004). Cell, 119, 591-602.

Bose I and Ghosh B. (2007). J Biosci, 32, 991-997.

Bottger A, Bottger V, Garcia-Echeverria C, Chene P, Hochkeppel HK, Sampson W, Ang K, Howard SF, Picksley SM and Lane DP. (1997). J Mol Biol, 269, 744-756.

Brooks CL and Gu W. (2003). Curr Opin Cell Biol, 15, 164-171.

Brooks CL and Gu W. (2008). Cancer Cell, 13, 377-378.

Brooks CL and Gu W. (2006). Mol Cell, 21, 307-315.

Brown CJ, Lain S, Verma CS, Fersht AR and Lane DP. (2009). Nat Rev Cancer, 9, 862-873.

Brummelkamp TR, Fabius AW, Mullenders J, Madiredjo M, Velds A, Kerkhoven RM, Bernards R and Beijersbergen RL. (2006). Nat Chem Biol, 2, 202-206.

Campisi J and Yaswen P. (2009). Aging Cell, 8, 221-225.

Canner JA, Sobo M, Ball S, Hutzen B, DeAngelis S, Willis W, Studebaker AW, Ding K, Wang S, Yang D and Lin J. (2009). Br J Cancer, 101, 774-781.

Cano A, Perez-Moreno MA, Rodrigo I, Locascio A, Blanco MJ, del Barrio MG, Portillo F and Nieto MA. (2000). Nat Cell Biol, 2, 76-83.

Canto C and Auwerx J. (2009). Trends Endocrinol Metab, 20, 325-331.

Choong ML, Yang H, Lee MA and Lane DP. (2009). Cell Cycle, 8, 2810-2818.

Chuikov S, Kurash JK, Wilson JR, Xiao B, Justin N, Ivanov GS, McKinney K, Tempst P, Prives C, Gamblin SJ, Barlev NA and Reinberg D. (2004). Nature, 432, 353-360.

Craig AL, Burch L, Vojtesek B, Mikutowska J, Thompson A and Hupp TR. (1999). Biochem J, 342 ( Pt 1), 133-141.

Ding K, Lu Y, Nikolovska-Coleska Z, Wang G, Qiu S, Shangary S, Gao W, Qin D, Stuckey J, Krajewski K, Roller PP and Wang S. (2006). J Med Chem, 49, 3432-3435.

DiTullio RA, Jr., Mochan TA, Venere M, Bartkova J, Sehested M, Bartek J and Halazonetis TD. (2002). Nat Cell Biol, 4, 998-1002.

Do TN, Ucisik-Akkaya E, Davis CF, Morrison BA and Dorak MT. (2009). Cancer Genet Cytogenet, 195, 31-36.

Downward J. (2003). Nat Rev Cancer, 3, 11-22.

Economopoulos KP and Sergentanis TN. (2009). Breast Cancer Res Treat.

Fotouhi N and Graves B. (2005). Curr Top Med Chem, 5, 159-165.

Galic V, Willner J, Wollan M, Garg R, Garcia R, Goff BA, Gray HJ and Swisher EM. (2007). Genes Chromosomes Cancer, 46, 239-247. 
Gorgoulis VG and Halazonetis TD. (2010). Curr Opin Cell Biol, 22, 816-827.

Grochola LF, Muller TH, Bond GL, Taubert H, Udelnow A and Wurl P. (2010b). Pancreas, 39, 76-80.

Grochola LF, Muller TH, Bond GL, Taubert H, Udelnow A and Wurl P. (2010a). Pancreas, 39, 76-80.

Gu W, Luo J, Brooks CL, Nikolaev AY and Li M. (2004). Novartis Found Symp, 259, 197-205.

Halaby MJ and Yang DQ. (2007). Gene, 395, 1-7.

Hasegawa K and Yoshikawa K. (2008). J Neurosci, 28, 8772-8784.

Haupt Y. (2004). Cell Cycle, 3, 884-885.

Hedstrom E, Eriksson S, Zawacka-Pankau J, Arner ES and Selivanova G. (2009). Cell Cycle, 8, 3576-3583.

Hohne MW, Halatsch ME, Kahl GF and Weinel RJ. (1992). Cancer Res, 52, 2616-2619.

Hollstein M, Moeckel G, Hergenhahn M, Spiegelhalder B, Keil M, Werle-Schneider G, Bartsch H and Brickmann J. (1998). Mutat Res, 405, 145-154.

Hollstein M, Rice K, Greenblatt MS, Soussi T, Fuchs R, Sorlie T, Hovig E, Smith-Sorensen B, Montesano R and Harris CC. (1994). Nucleic Acids Res, 22, 3551-3555.

Horiguchi K, Shirakihara T, Nakano A, Imamura T, Miyazono K and Saitoh M. (2009). J Biol Chem, 284, 245-253.

Hruban RH, Goggins M and Kern SE. (1999). Curr Opin Gastroenterol, 15, 404-409.

Hruban RH, Goggins M, Parsons J and Kern SE. (2000). Clin Cancer Res, 6, 2969-2972.

Hruban RH, Maitra A, Kern SE and Goggins M. (2007). Gastroenterol Clin North Am, 36, 83149, vi.

Ito A, Kawaguchi Y, Lai CH, Kovacs JJ, Higashimoto Y, Appella E and Yao TP. (2002). $E M B O ~ J, 21,6236-6245$.

Iwakuma T and Lozano G. (2003). Mol Cancer Res, 1, 993-1000.

Jemal A, Siegel R, Xu J and Ward E. (2010). CA Cancer J Clin, 60, 277-300.

Kalthoff H, Schmiegel W, Roeder C, Kasche D, Schmidt A, Lauer G, Thiele HG, Honold G, Pantel K, Riethmuller G and . (1993). Oncogene, 8, 289-298.

Klein C and Vassilev LT. (2004). Br J Cancer, 91, 1415-1419.

Koblish HK, Zhao S, Franks CF, Donatelli RR, Tominovich RM, LaFrance LV, Leonard KA, Gushue JM, Parks DJ, Calvo RR, Milkiewicz KL, Marugan JJ, Raboisson P, Cummings MD, Grasberger BL, Johnson DL, Lu T, Molloy CJ and Maroney AC. (2006). Mol Cancer Ther, 5, 160-169.

Kruse JP and Gu W. (2009). Cell, 137, 609-622.

Laghi L, Orbetegli O, Bianchi P, Zerbi A, Di C, V, Boland CR and Malesci A. (2002). Oncogene, 21, 4301-4306.

Lahav G. (2008). Adv Exp Med Biol, 641, 28-38.

Lain S, Hollick JJ, Campbell J, Staples OD, Higgins M, Aoubala M, McCarthy A, Appleyard V, Murray KE, Baker L, Thompson A, Mathers J, Holland SJ, Stark MJ, Pass G, Woods J, Lane DP and Westwood NJ. (2008). Cancer Cell, 13, 454-463.

Lang A, Palmeback WP and Wingren S. (2009). Oncol Rep, 22, 575-579.

Lang GA, Iwakuma T, Suh YA, Liu G, Rao VA, Parant JM, Valentin-Vega YA, Terzian T, Caldwell LC, Strong LC, El-Naggar AK and Lozano G. (2004). Cell, 119, 861-872.

Lee JT and Gu W. (2010). Cell Death Differ, 17, 86-92.

Lee SH, Lee SJ, Jung YS, Xu Y, Kang HS, Ha NC and Park BJ. (2009a). Neoplasia, 11, 22-31, 6p. 
Lee SH, Shen GN, Jung YS, Lee SJ, Chung JY, Kim HS, Xu Y, Choi Y, Lee JW, Ha NC, Song GY and Park BJ. (2010a). Oncogene, 29, 4576-4587.

Lee SH, Shen GN, Jung YS, Lee SJ, Chung JY, Kim HS, Xu Y, Choi Y, Lee JW, Ha NC, Song GY and Park BJ. (2010b). Oncogene, 29, 4576-4587.

Lee SJ, Jung YS, Lee SH, Chung HY and Park BJ. (2009b). Int J Oncol, 34, 1637-1643.

Lehman JA, Eitel JA, Batuello CN and Mayo LD. (2008). Expert Opin Drug Discov, 3, 13091321.

Lind H, Zienolddiny S, Ekstrom PO, Skaug V and Haugen A. (2006). Int J Cancer, 119, 718721.

Mancini F, Conza GD and Moretti F. (2009a). Curr Genomics, 10, 42-50.

Mancini F, Di CG, Pellegrino M, Rinaldo C, Prodosmo A, Giglio S, D'Agnano I, Florenzano F, Felicioni L, Buttitta F, Marchetti A, Sacchi A, Pontecorvi A, Soddu S and Moretti F. (2009b). EMBO J, 28, 1926-1939.

Marigo V, Johnson RL, Vortkamp A and Tabin CJ. (1996). Dev Biol, 180, 273-283.

Mendrysa SM, McElwee MK, Michalowski J, O'Leary KA, Young KM and Perry ME. (2003). Mol Cell Biol, 23, 462-472.

Millau JF, Bastien N and Drouin R. (2009). Mutat Res, 681, 118-133.

Ohmiya N, Taguchi A, Mabuchi N, Itoh A, Hirooka Y, Niwa Y and Goto H. (2006a). J Clin Oncol, 24, 4434-4440.

Ohmiya N, Taguchi A, Mabuchi N, Itoh A, Hirooka Y, Niwa Y and Goto H. (2006b). J Clin Oncol, 24, 4434-4440.

Okamoto K, Taya Y and Nakagama H. (2009). FEBS Lett, 583, 2710-2714.

Olive KP, Tuveson DA, Ruhe ZC, Yin B, Willis NA, Bronson RT, Crowley D and Jacks T. (2004). Cell, 119, 847-860.

Patel S and Player MR. (2008). Expert Opin Investig Drugs, 17, 1865-1882.

Pellegata NS, Sessa F, Renault B, Bonato M, Leone BE, Solcia E and Ranzani GN. (1994). Cancer Res, 54, 1556-1560.

Perfumo C, Parodi S, Mazzocco K, Defferrari R, Inga A, Scarra GB, Ghiorzo P, Haupt R, Tonini GP and Fronza G. (2009). Pediatr Blood Cancer, 53, 576-583.

Schumacher B and Gartner A. (2006). Future Oncol, 2, 145-153.

Secchiero P, di Iasio MG, Gonelli A and Zauli G. (2008). Curr Pharm Des, 14, 2100-2110.

Shangary S, Qin D, McEachern D, Liu M, Miller RS, Qiu S, Nikolovska-Coleska Z, Ding K, Wang G, Chen J, Bernard D, Zhang J, Lu Y, Gu Q, Shah RB, Pienta KJ, Ling X, Kang S, Guo M, Sun Y, Yang D and Wang S. (2008a). Proc Natl Acad Sci U S A, 105, 39333938.

Shangary S, Qin D, McEachern D, Liu M, Miller RS, Qiu S, Nikolovska-Coleska Z, Ding K, Wang G, Chen J, Bernard D, Zhang J, Lu Y, Gu Q, Shah RB, Pienta KJ, Ling X, Kang S, Guo M, Sun Y, Yang D and Wang S. (2008b). Proc Natl Acad Sci U S A, 105, 39333938.

Shangary S and Wang S. (2009). Annu Rev Pharmacol Toxicol, 49, 223-241.

Shinohara A, Sakano S, Hinoda Y, Nishijima J, Kawai Y, Misumi T, Nagao K, Hara T and Matsuyama H. (2009). Cancer Sci.

Sohn TA. (2002). Minerva Chir, 57, 561-574.

Somasundaram K. (2000). Front Biosci, 5, D424-D437.

Stommel JM and Wahl GM. (2005). Cell Cycle, 4, 411-417.

Vassilev LT. (2004a). Cell Cycle, 3, 419-421. 
Vassilev LT. (2005). J Med Chem, 48, 4491-4499.

Vassilev LT. (2004b). Cell Cycle, 3, 419-421.

Vassilev LT. (2007). Trends Mol Med, 13, 23-31.

Vassilev LT, Vu BT, Graves B, Carvajal D, Podlaski F, Filipovic Z, Kong N, Kammlott U, Lukacs C, Klein C, Fotouhi N and Liu EA. (2004). Science, 303, 844-848.

Wang Z, Li Y, Ahmad A, Banerjee S, Azmi AS, Kong D and Sarkar FH. (2011). Nat Rev Gastroenterol Hepatol, 8, 27-33.

Watanabe T, Hotta T, Ichikawa A, Kinoshita T, Nagai H, Uchida T, Murate T and Saito H. (1994). Blood, 84, 3158-3165.

Watanabe T, Ichikawa A, Saito H and Hotta T. (1996). Leuk Lymphoma, 21, 391-7, color.

Whitcomb D and Greer J. (2009). Clin Gastroenterol Hepatol, 7, S29-S34.

White AC, Tran K, Khuu J, Dang C, Cui Y, Binder SW and Lowry WE. (2011). Proc Natl Acad Sci U S A, 108, 7425-7430.

Wiederschain D, Gu J and Yuan ZM. (2001). J Biol Chem, 276, 27999-28005.

Yook JI, Li XY, Ota I, Hu C, Kim HS, Kim NH, Cha SY, Ryu JK, Choi YJ, Kim J, Fearon ER and Weiss SJ. (2006). Nat Cell Biol, 8, 1398-1406.

Zauli G, di Iasio MG, Secchiero P, Dal BM, Marconi D, Bomben R, Del PG and Gattei V. (2009). Curr Cancer Drug Targets, 9, 510-518.

Zhang Z and Zhang R. (2005). Curr Cancer Drug Targets, 5, 9-20. 


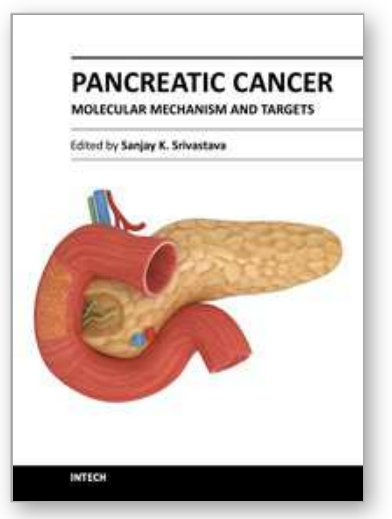

\author{
Pancreatic Cancer - Molecular Mechanism and Targets \\ Edited by Prof. Sanjay Srivastava
}

ISBN 978-953-51-0410-0

Hard cover, 432 pages

Publisher InTech

Published online 23, March, 2012

Published in print edition March, 2012

This book provides the reader with an overall understanding of the biology of pancreatic cancer, hereditary, complex signaling pathways and alternative therapies. The book explains nutrigenomics and epigenetics mechanisms such as DNA methylation, which may explain the etiology or progression of pancreatic cancer. Book also summarizes the molecular control of oncogenic pathways such as K-Ras and KLF4. Since pancreatic cancer metastasizes to vital organs resulting in poor prognosis, special emphasis is given to the mechanism of tumor cell invasion and metastasis. Role of nitric oxide and Syk kinase in tumor metastasis is discussed in detail. Prevention strategies for pancreatic cancer are also described. The molecular mechanisms of the anti-cancer effects of curcumin, benzyl isothiocyante and vitamin $D$ are discussed in detail. Furthermore, this book covers the basic mechanisms of resistance of pancreatic cancer to chemotherapy drugs such as gemcitabine and 5 -flourouracil.

\title{
How to reference
}

In order to correctly reference this scholarly work, feel free to copy and paste the following:

Asfar S. Azmi, Minsig Choi and Ramzi M. Mohammad (2012). p53 Re-Activating Small Molecule Inhibitors for the Treatment of Pancreatic Cancer, Pancreatic Cancer - Molecular Mechanism and Targets, Prof. Sanjay Srivastava (Ed.), ISBN: 978-953-51-0410-0, InTech, Available from:

http://www.intechopen.com/books/pancreatic-cancer-molecular-mechanism-and-targets/emerging-smallmolecule-inhibitors-for-the-treatment-of-pancreatic-cancer

\section{INTECH}

open science | open minds

\section{InTech Europe}

University Campus STeP Ri

Slavka Krautzeka 83/A

51000 Rijeka, Croatia

Phone: +385 (51) 770447

Fax: +385 (51) 686166

www.intechopen.com

\section{InTech China}

Unit 405, Office Block, Hotel Equatorial Shanghai

No.65, Yan An Road (West), Shanghai, 200040, China

中国上海市延安西路 65 号上海国际贵都大饭店办公楼 405 单元

Phone: +86-21-62489820

Fax: $+86-21-62489821$ 
(C) 2012 The Author(s). Licensee IntechOpen. This is an open access article distributed under the terms of the Creative Commons Attribution 3.0 License, which permits unrestricted use, distribution, and reproduction in any medium, provided the original work is properly cited. 